Nutr. Dieta 1961;3:169

\title{
Zusammenhänge zwischen Arteriosklerose und Ernährung
}

Referate und Diskussionen eines Kolloquiums im Rahmen des VI. Intemationalen Kongresses für Erkrankungen der Thoraxorgane Wien, Universität, 30. August 1960

Eínleitung

Herr Prof. Dr. K. Fellinger, Wien Herausgegeben von Herrn Dozent Dr. R. Wenger, Wien (Leiter des

Kolloquiums)

Meine sehr geehrten Damen und Herren!

Erlauben Sie mir, daß ich Sie, auch im Namen des Präsidenten des VI. Intemationalen Kongresses für Erkrankungen der Thoraxorgane, Herrn Prof. Dr. K. Fellinger, aufrichtig begrüße und Ibnen dafür danke, daß Sie unserer Einladung folgten, zu dem Kolloquium über «Arteriosklerose und Ernährung» nach Wien zu kommen und über Ihre eigenen Forschungsergebnisse zu berichten.

Dieser Fragenkomplex steht derzeit auch stark im Brennpunkt des Interesses weiter Kreise und wird vielfach auch in der Presse be-handelt, was sicherlich nicht immer zum Vorteil des wissenschaft-lichen Fortschritts ist. Außerdem sind die damit zusammenhängenden Fragen in mancher Hinsicht mit wirtschaftlichen Interessen ver-schiedenster Zweige der Volkswirtschaft verkniipft, so daß es gerade auch auf diesem Gebiete notwendig ist, zu starke Beeinflussungen der Forscher von außen her abzuwehren.

Das Kolloquium wird, mit mehr theoretischen Forschungsberichten beginnend, zu praktischdiätetischen Schlußfolgerungen fortschreiten, wobei Diskussionsbemerkungen zu jedem der Kurzberichte erwünscht und erbeten sind. Ich darf Sie somit einladen, mit Ihren Forschungsberichten zu beginnen.

R. Wenger

Wien

Nutritio et Dieta, Vol. 3, No. 3 (1961) 